Brain MRI has a distinct feature with eye-of the-tiger sign characterized by bilateral hypointensity of the globus pallidus with a central area of hyperintensity on T2-weighted images. Peripheral blood acanthocytosis and low plasma pre beta lipoprotein are supportive evidence. Establishing PANK 2 variant on molecular genetic testing is confirmatory.

Management is mainly supportive. Dystonia can be controlled with benzodiazepines, anticholinergics and botulinum toxin. Spasticity can be revealed with baclofen. Stereotactic surgical modalities such as thalamotomy, pallidotomy and deep brain stimulation of the globus pallidus can help to control symptoms.

We present a 5year old boy who presented with tiptoe walking who gradually deteriorated neurodevelopmentaly. $\mathrm{He}$ had declined in academic performance and progressive dystonia, dysarthria and spasticity was there. He had hyperreflexia but parkinsonism, chorea or myoclonus was not evident. The neuro-ophthalmologic examination was normal. Laboratory work up was normal including serum copper, ceruloplasmin levels. Blood smear had no acanthocytes. T2 weighted brain MRI was consistent with 'eye-of the-tiger' sign. Genetic testing was not performed due to the cost effect.

The child is under multidisciplinary rehabilitation to improve quality of life.

This case report highlights the importance of considering this entity in progressive dystonia with neurodegeneration.

\section{PEDIATRIC DEMYELINATING DISORDERS - A SINGLE CENTRE EXPERIENCE IN A TERTIARY CARE CENTRE, SRI LANKA}

Thambiliyagodage Manori Prasadani ${ }^{\star}$. Postgraduate Institute of Medicine University of Colombo

\subsection{6/archdischild-2021-repch.868}

Background Pediatric demyelinating disorders (PDDs) are rare neurological conditions reported among children. It has variable neurological presentations which challenge in diagnosis.

Objectives This study was conducted to assess the clinical characteristics and outcomes of the PDDs reported to paediatric neurology unit, Teaching Hospital Karapitiya (THK).

Methods A cross sectional study was conducted among neurological symptomatic patients attended, paediatric neurology unit at THK for five years duration. Data was extracted from clinical records available at paediatric neurology unit. All patients reported during study period were included.

Results A total sample of 9245 children with neurological symptoms were reported during the study period. Among them, 20 children were diagnosed to have PDDs with $0.21 \%$ of five years period prevalence. Acute Demyelinating Encephalomyelitis (ADEM) $(\mathrm{n}=14,70.0 \%)$ \& Optic neuritis $(n=4,20.0 \%)$ were identified as common PDDs while Transverse myelitis and Neuromyelitis Optica spectrum of disorders ( $n=1,10.0 \%$ for both) were identified as less common. No patient was identified with Multiple sclerosis. Half of the children with PDDs were male $(n=10)$. Mean (SD) age of the presentation of PDDS was 9.1(3.0) years. Altered consciousness $(n=12,85.7 \%)$, headache $(n=10$, $71.4 \%)$ sensory deficits $(n=8,57.2 \%)$, convulsions $(n=6$,
42.8\%) and acute psychiatric symptoms $(n=5,35.7 \%)$ were identified as common presentation for ADEM. All the children with Optic neuritis presented with reduced visual acuity. Blood investigations, viral isolation and neuroimaging were non-significant in all the children except who were diagnosed with ADEM. Majority of the children completely recovered following treatment $(85.7 \%, \mathrm{n}=12$ for ADEM and $75.0 \%, \mathrm{n}=3$ for Optic neuritis). However, one death and one residual defect was reported among ADEM patients while urinary and bowel incontinence was reported for Transverse myelitis.

Conclusions PDDs were rare among neurological symptomatic patients attended to pediatric neurology unit at THK. Clinical presentation, investigations, neuroimaging findings varied among them challenging the diagnosis. Majority had better outcome following treatment.

\section{An Introduction to Sustainable Healthcare and the RCPCH Climate Change Programme}

\section{CARBON LITERACY TRAINING IN HEALTHCARE PROFESSIONALS: A SURVEY OF PAEDIATRICIANS}

Kate Macfarlane* . Alder Hey Childrens Hospital

\subsection{6/archdischild-2021-rcpch.869}

Background The NHS is responsible for 5\% of the UK's carbon footprint. Every professional within the NHS has a duty to commit to reducing their department's carbon emissions to meet the government's net zero pledge by 2040 .

As Paediatricians and advocates of children, young people and future generations, it is our responsibility to take an active role in tackling the climate emergency. The first step is education and carbon literacy of our current workforce. Carbon Literacy' is defined the awareness of climate change and the climate impacts of mankind's everyday actions.

In October 2020, The RCPCH joined a growing coalition of public health and medical organisations in declaring a climate emergency. This involved the convening of a Climate Change Working Group with workstreams including, investment policy, greening the workplace, research and curriculum changes.

As Paediatricians, educators, leaders and mentors, we have a major role in influencing trusts to implement Green Plans and carbon reduction strategies. Through education of health care professionals and ensuring that sustainability is at the heart of every quality improvement activity we can work with governments towards the net zero pledge.

Objectives

- Investigate current views on the importance of the NHS's role in the climate crisis within health professionals.

- To assess prevalence of previous carbon literacy training, awareness and motivation to participate in further training.

Methods The author developed a survey of 9 questions enquiring about previous carbon literacy training, quality improvement projects involving sustainability and perceived importance of the climate crisis within the NHS. The survey was distributed by SurveyMonkey to all Paediatric Doctors in 
the Mersey Deanery training group and those working on Paediatric Intensive Care. The survey was anonymous and open between February and March 2021 and there was no financial incentive to participate.

Results 38 healthcare professionals responded over a 1-month period. They were all Doctors and all except 1 were Paediatricians (Trainees, Consultants and Staff Grades). Respondents' graduation dates ranged from 1987 to 2016 and they reported attending Medical Schools both in the UK and Internationally. Only $2(5 \%)$ of the participants reported formal Carbon Literacy training, while 80\% (30) reported that they would not know how to calculate the carbon footprint of their personal or departmental activities. 95\% (36) said they would be interested in knowing more about how to reduce the carbon footprint of the NHS.
Conclusions This survey highlights that health professionals are very interested and invested in the climate emergency but the majority of them have not many have had formal carbon literacy training. By incorporating sustainability teaching into the curriculum, Paediatricians and those working in our specialty will feel more empowered to make changes to their quality improvement projects, lobby trusts and work together in driving down the carbon footprint of our departments and those of the future. This survey is an opportunity to engage interested professionals and will be extended to other health professionals in our region. It will act as a guide to curriculum change and educational interventions on climate change. 